\section{Polyploidization of glia in neural development links tissue growth to blood-brain barrier integrity}

\author{
Yingdee Unhavaithaya and Terry L. Orr-Weaver ${ }^{1}$
}

Whitehead Institute, Department of Biology, Massachusetts Institute of Technology, Cambridge, Massachusetts 02142, USA

Proper development requires coordination in growth of the cell types composing an organ. Many plant and animal cells are polyploid, but how these polyploid tissues contribute to organ growth is not well understood. We found the Drosophila melanogaster subperineurial glia (SPG) to be polyploid, and ploidy is coordinated with brain mass. Inhibition of SPG polyploidy caused rupture of the septate junctions necessary for the blood-brain barrier. Thus, the increased SPG cell size resulting from polyploidization is required to maintain the SPG envelope surrounding the growing brain. Polyploidization likely is a conserved strategy to coordinate tissue growth during organogenesis, with potential vertebrate examples.

Supplemental material is available for this article.

Received August 19, 2011; revised version accepted November 23, 2011.

The coordination of growth between different tissues is crucial to achieve proper development and is particularly important during organogenesis. The growth of distinct tissues forming an organ must be scaled to size, but it is not clear how this is attained. Tissue growth can be controlled by regulation of cell division, apoptosis, or cell size (Crickmore and Mann 2008; Breuninger and Lenhard 2010). Although increased cell size can be achieved via accumulation of cell mass during growth of diploid cells, increased cell size often is produced during development via polyploidization (Edgar and Orr-Weaver 2001; Lee et al. 2009). In plants, the size of many structures such as trichromes and root hairs is dictated by the ploidy of progenitor cells. Larval growth in Drosophila melanogaster is the result of increased ploidy of most larval organs. In vertebrates, polyploidization of the megakaryocytes and trophoblasts ensures the cells are large enough for their functions in platelet production and the placental barrier, respectively (Edgar and Orr-Weaver 2001; Lee et al. 2009). How overall ploidy is determined is not known, and it is not understood how the sizes of polyploid tissue layers are scaled with proliferating diploid cells in organogenesis.

[Keywords: cell size; Drosophila; nervous system development; polyploid; tissue growth]

${ }^{1}$ Corresponding author.

E-mail weaver@wi.mit.edu.

Article is online at http://www.genesdev.org/cgi/doi/10.1101/gad.177436.111.

Freely available online through the Genes \& Development Open Access option.
One mechanism by which cells polyploidize is via a modified cell cycle, termed the endocycle, in which DNA replication alternates with an intervening unique gap phase without mitosis or cell division (Smith and Orr-Weaver 1991). This process, also termed endoreplication or endoreduplication, does not require the mitotic regulators. Although the endocycle is used throughout Drosophila larval tissues and plants, in vertebrates, some polyploid tissues undergo some aspects of mitosis. Mammalian hepatocytes undergo mitosis and nuclear division but not cytokinesis (Styles 1993), resulting in multinucleated polyploid cells. In megakaryocytes, anaphase A but not anaphase B occurs, giving rise to polyploid cells with a single nucleus (Nagata et al. 1997). Here, we refer to these cell cycle variations as endomitosis.

Potential developmental roles for polyploidy in the nervous system are largely unexplored. The nervous system is made up mainly of two types of cells: neurons and glia. In the Drosophila nervous system (Fig. 1A), the glia are likely to be polyploid, as large glia cells with large nuclei have been observed (Banerjee and Bhat 2007; Silies et al. 2007; Stork et al. 2008). Nuclear size and ploidy are tightly linked (Edgar and Orr-Weaver 2001), suggesting that the observed cells could be polyploid. Consistent with this idea, Prokop and Technau (1991) observed that a population of glia undergoes endoreplication. However, the extent and the function of polyploidy in glia cells are not well understood.

Here, we report that the subperineurial glia (SPG) are polyploid and that polyploidy is critical for the maintenance of the blood-brain barrier. We propose that polyploidy in the SPG plays an important role to increase cell size to maintain the integrity of the blood-brain barrier as the underlying neuronal mass increases during larval brain development.

\section{Results and Discussion}

\section{The SPG are polyploid}

To identify polyploid glia cells, we examined the DNA content of fixed adult Drosophila brain samples in which nervous system development was complete (Technau 2008). We observed that a fraction of nuclei had high amounts of DNA and was positive for the glia-specific Repo protein (Xiong et al. 1994), consistent with some glia nuclei being polyploid (Supplemental Fig. 1A). To verify the ploidy of glia cells, we carried out FACS of nuclei from repo $>$ gal4/+;UAS $>g f p^{n l s} /+$ larval brains, a marking system in which the yeast GAL4 activator drives expression of nuclear GFP in all glia. When compared with the $U A S>g f p^{n l s} /+$ control sample, the GFP-positive nuclei FACS profile from the repo $>$ gal4/+;UAS $>g f p^{\text {nls }} /+$ nuclei was enriched for 4C (twice the diploid genomic content), and peaks of higher DNA content were present (Supplemental Fig. 1B). This indicated that a subpopulation of glia is polyploid. The position of the aforementioned polyploid glia on the exterior of the brain lobes suggested that they were SPG. The SPG envelop the neurons and form a seal through their septate junctions, the Drosophila equivalent of tight junctions, which constitute the bloodbrain barrier (Fig. 1A; Stork et al. 2008; Desalvo et al. 2011; Hatan et al. 2011). 
A

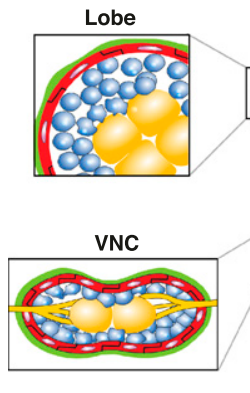

B

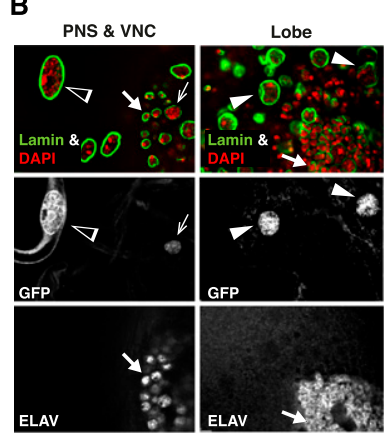

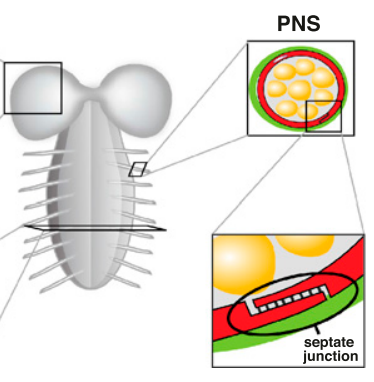

C

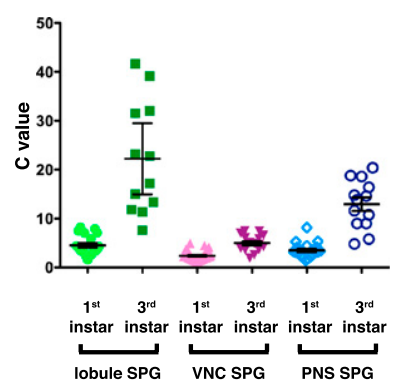

Figure 1. SPG are polyploid. (A) Schematic of the Drosophila larval nervous system organization. (Lobe) Brain lobe. SPG (red) are connected via septate junctions and form an envelope around the neurons (blue) in the cortex surrounding the neuropil (orange). Perineurial glia and the neural lamella (both depicted in green) cover the entire nervous system. (B) SPG nuclei from the PNS (open arrow heads), VNC (barbed arrows), and brainlobes (closed arrow heads) of moody $>$ gal4(II)/+;UAS $>g f p^{n l s} /+$ third instar nervous system are shown. SPG are marked by $\mathrm{GFP}^{\text {nls }}$ via the SPGspecific moody driver. A high DNA content (DAPI, red) is seen in GFP-marked SPG nuclei (outlined by nuclear envelope component lamin, green), compared with the DNA content of the diploid neuronal nuclei marked by ELAV (arrows). (C) Scatter plot showing the mean and $95 \%$ confidence interval of SPG ploidy at different locations in the nervous system. Each data point is total ploidy from one cell. ( $Y$-axis) $\mathrm{C}$ value; $(X$-axis) category of SPG. In the VNC, the thoracic SPG are larger and have up to twofold higher ploidy than the abdominal SPG.

To test whether the observed polyploid glia were SPG, we specifically labeled SPG using GAL4 under the control of the regulatory elements for moody, a gene encoding $G$ protein-coupled receptors expressed specifically in the SPG, to drive nuclear GFP ${ }^{\text {nls }}$ under UAS control (Fig. 1B,C; Bainton et al. 2005). The DAPI intensity of individual SPG nuclei was measured in larvae and normalized against the adjacent Elav-positive, post-mitotic, diploid neurons (Robinow and White 1991). Different levels of SPG ploidy were observed for different SPG categories, which were grouped according to their location on the nervous system (Fig. 1). In the brain, these polyploid SPG were either mononucleate or multinucleate (see below). The average $\mathrm{C}$ values (the haploid genome content) of the first instar larval brain lobe SPG, ventral nerve cord (VNC) SPG, and peripheral nervous system (PNS) SPG were 4.5C, 2.4C, and 3.5C, respectively (Fig. 1C). SPG ploidy increased significantly as the larvae developed to the third instar stage, with the average $\mathrm{C}$ value of the lobe SPG, VNC SPG, and PNS SPG at 22.2C, 5C, and 15C, respectively. The increased ploidy levels correlated with increased cell size (Supplemental Fig. 2). These data confirm that SPG are polyploid and reveal that ploidy levels vary depending on domains of the nervous system.

\section{Both endoreplication and endomitosis contribute to SPG polyploidy}

To analyze the mechanism by which the SPG polyploidize, as well as the developmental timing, we asked whether any cell division occurs in the SPG during larval development. This was addressed by (1) marking the boundaries of the SPG cells with a Neurexin IV::GFP fusion protein that localizes to septate junctions (Edenfeld et al. 2006), (2) specifically labeling the SPG nuclei via moody $>$ gal4-driven $\mathrm{GFP}^{\text {nls }}$, and (3) counting cell number during development (Supplemental Movie 1A,B). The number of SPG cells in the brain lobe stayed constant throughout larval development, with $13.76 \pm 0.9$ and $13.83 \pm 0.7$ cells for first and third instar larvae, respectively. This result indicated that brain lobe SPG do not undergo cytokinesis during larval development.

We next monitored when DNA replication occurred in the SPG by using the nucleotide analog EdU to label brains that expressed SPG-specific GFP nls (Fig. 2A-C). SPG initiated DNA replication within $20 \mathrm{~h}$ of larval hatching from the embryo (Supplemental Table 1). The percent of SPG undergoing S phase varied between regions of the nervous system for each developmental time point, with replication continuing longest in the VNC (Supplemental Table 1).

Unexpectedly, in addition to the polyploid mononucleate SPG, we also observed multinucleate polyploid cells in the brain lobes (Fig. 2D,E). Thirty percent of SPG were

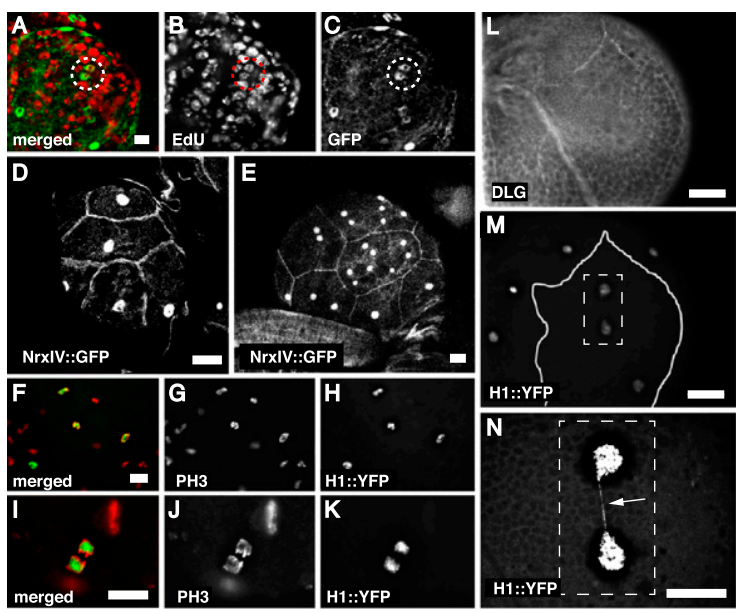

Figure 2. SPG cells of the brain lobes use different strategies to polyploidize. SPG were labeled via GFP ${ }^{\text {nls }}$ driven by the moody>gal4(II) SPG-specific driver. S phase was detected by EdU incorporation. Chromosome segregation in SPG was monitored via H1::YFP, specifically expressed in SPG via a moody>gal4(II) driver transgene and by PH3 antibody staining. $(A-C)$ EdU was detected in the SPG of first instar larval brain lobes. $(A)$ Merged. $(B)$ EdU. $(C)$ GFP. $(D, E)$ Cell boundaries and nuclear number in the SPG. The cell boundaries were marked with a NeurexinIV::GFP. Nuclei were marked by GFP ${ }^{\text {nls }}$, driven by moody $>$ gal4(II). First instar larval brain lobe $(D)$ and third instar larval brain lobe $(E)$. $(F-H)$ Asynchronous mitotic figures were observed in SPG in third instar larval brain lobes. $(G)$ PH3. $(H)$ H1:: YFP, exclusive in SPG. (I-K) Anaphase cells were observed. (J) PH3, (K) H1::YFP, exclusive in SPG. (N) A magnification of the dashed rectangular area in $M$ shows two H1::YFP-marked SPG nuclei. The solid line in $M$ depicts the cell boundary of the SPG marked by staining with antibody to Discs Large in L. A chromosome bridge was detected via $\mathrm{H1}::$ YFP (white arrow). $N$ is overexposed so that the chromosome bridge can clearly be seen. Bars, $20 \mu \mathrm{m}$. 
mononucleated, compared with $70 \%$ multinucleated $(n=$ 25 ) in the brain, whereas in the ventral cord and PNS, all of the polyploid SPG were mononucleate. The presence of multinucleate SPG in the brain lobes predicted that mitosis occurred in these cells. This was tested by staining with an antibody against the mitosis-specific marker phosphorylated histone $\mathrm{H} 3$ (PH3). In this experiment, the SPG were labeled with a histone $\mathrm{H} 1:$ YFP fusion protein expressed solely in the SPG, permitting the cells to be identified even after nuclear envelope breakdown (Pinnola et al. 2007). Mitotic figures were observed in the brain lobe SPG (Fig. 2F-K; Supplemental Table 2). These multiple mitotic nuclei were present within single cells and frequently were connected by chromosome bridges, a hallmark of defective DNA replication and/or abnormal chromosome segregation (Fig. 2L-N; Strunnikov 2010). Chromosome bridges were detected in five out of 18 brains examined. Taken together, these developmental studies show that the SPG do not increase in cell number during larval development, but rather become polyploid. In the VNC and PNS, this appears to occur solely by the endocycle, whereas in the brain lobe, many SPG do nuclear division but not cytokinesis. The reason for these regional differences in the SPG remains to be determined.

SPG polyploidy is required for a functional blood-brain barrier

We examined whether increased ploidy was required for SPG function by ablating DNA replication in the SPG during the period of endoreplication and endomitosis. To achieve this, we specifically depleted the cells for a protein required for the initiation of DNA replication: the Cdt1/Dup component of the prereplication complex at replication origins (Whittaker et al. 2000; Bell and Dutta 2002). We decreased $c d t 1 / d u p$ in the SPG by driving expression of $U A S>c d t 1$ (dup) dsRNA via the transgene moody>gal4(II). We verified that the $c d t 1$ (dup)RNAi knockdown was effective, judged by undetectable levels of Dup in the SPG $(n=72)$ and decreased EdU incorporation in the $c d t 1 / d u p-a b-$ lated SPG compared with the control $(n=120)$ (Fig. 3A). Consistent with the relationship between ploidy and cell size, inhibition of polyploidy in the SPG resulted in a cell size decrease (Fig. 4B).

A key function of the SPG is to ensure the blood-brain barrier, maintained by septate (tight) junctions between the SPG (Stork et al. 2008; Hatan et al. 2011). The blood-brain barrier is necessary for ionic homeostasis between the brain and the hemolymph (Banerjee and Bhat 2007). To ask whether SPG polyploidy is required for the function of the blood-brain barrier, we performed dye injection assays (Bainton et al. 2005; Schwabe et al. 2005) on animals in which polyploidization of the SPG was blocked. For these experiments, three different drivers were used for cdt1(dup)RNAi in the SPG: moody >gal4 (II), moody>gal4 (III), and MZ97>gal4. Tetrarhodamine dextran $(10 \mathrm{kDa})$ dye was injected into the body cavity of the larvae, and dye penetration into the brain was examined. In the cdt1(dup)RNAi uninduced control as well as in the three SPG drivers alone, the dye did not penetrate the blood-brain barrier (Fig. 3B). In contrast, when polyploidization was reduced by ablation of $c d t 1$ (dup) specifically in the SPG, dye penetration was detectable as fluorescence around the neuroblasts and outlining the neuropil (Fig. 3B; quantified in Supplemental Table 3). The dye penetration defect was observed with all three SPG-specific GAL4 driver lines in combination with $U A S>c d t 1$ (dup)RNAi (Fig. 3B), arguing that this defect is due to the loss of $c d t 1$ (dup) function and is not caused by the insertion of the SPG GAL4 driver in the genome.

We then asked whether septate junctions themselves were defective. The patterns of the septate junction components Neurexin IV (NrxIV) (Baumgartner et al. 1996) and Discs Large (Dlg) (Woods and Bryant 1991) were abnormal in all of the brain samples with cdt1(dup)ablated SPG $[n=97$ (NrxIV and Dlg) in MZ97>gal4/+; $U A S>\operatorname{cdt} 1$ (dup)RNAi/+; $n=57$ (NrxIV) and $n=35$ (Dlg) in moody>gal4(II)/+;UAS>cdt1(dup)RNAi/+] (Fig. 3C). Disruption of septate junctions by reduction of polyploidization was confirmed by inhibiting DNA replication via an alternate method: pcna RNAi in the SPG (Supplemental
A

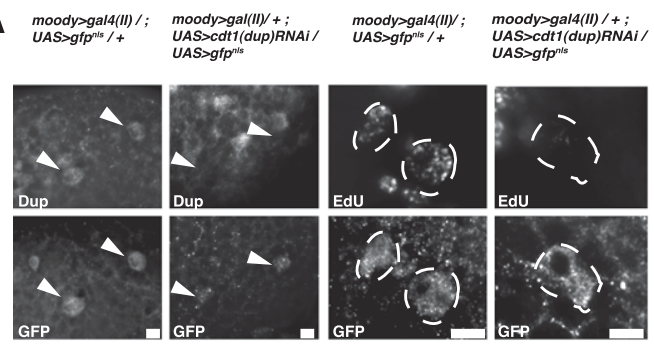

B
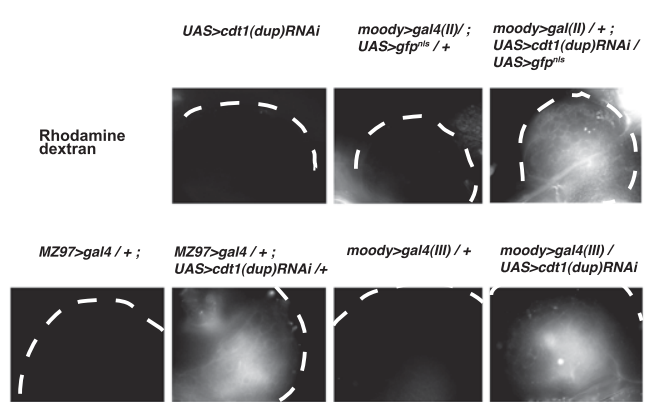

Figure 3. Inhibition of SPG polyploidy disrupts the blood-brain barrier. $(A)$ Inhibition of DNA replication in SPG. Dup protein expression and EdU incorporation were greatly diminished in the third instar brain after cdt1(dup) ablation by moody>gal4(II)/+; $U A S>c d t 1$ (dup)/UAS $>g f p^{n l s}$ RNAi. Large arrowheads indicate the position of the GFPpositive SPG nuclei. The dotted lines outline each nucleus in the EdU labeling experiment. Bars, $5 \mu \mathrm{m}$. (B) Breached blood-brain barrier in replication-blocked SPG. The dotted lines show the edge of the brain lobe. When $c d t 1$ (dup) was ablated in the SPG by moody>gal4(II)/+; $U A S>c d t 1$ (dup)RNAi/UAS $>$ gfp $p^{n l s}$, MZ97>gal4/+;UAS $>$ cdt1(dup)RNAi/+, or moody $>$ gal4(III)/ $U A S>c d t 1$ (dup)RNAi, the injected dye penetrated the brain. In contrast, there is no dye signal

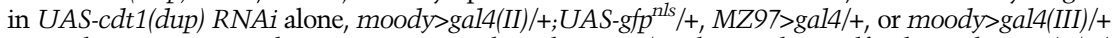
controls. M797 is another gene expressed in the SPG (Beckervordersandforth et al. 2008). (C) Blocking replication in the SPG caused a defective septate junction phenotype. Disrupted NrxIV and Dlg staining patterns in moody $>$ gal4(II)/+;UAS $>$ cdt1(dup)RNAi/+ compared with normal pattern in controls [UAS $>$ cdt1 1 (dup)RNAi and moody $\left.>g a l 4(I I) /+; U A S>g f p^{n l s} /++\right]$. Arrows indicate disrupted septate junctions. Bars, $10 \mu \mathrm{m}$. 
A

A MZ97>gal 4/+; MZ97>gal4/+

$U A S>g f p^{n / s} /+\quad$ UAS $>$ cdt1 $1(d+p)$
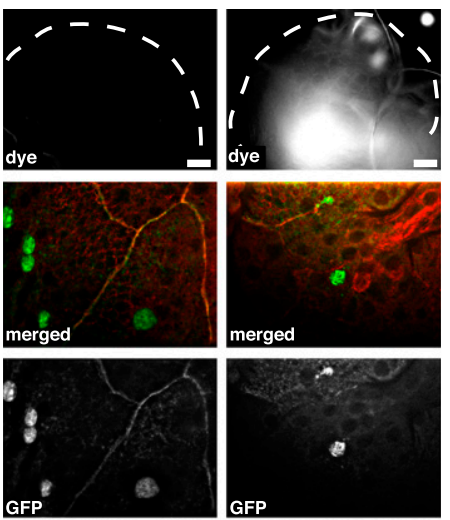

DLG
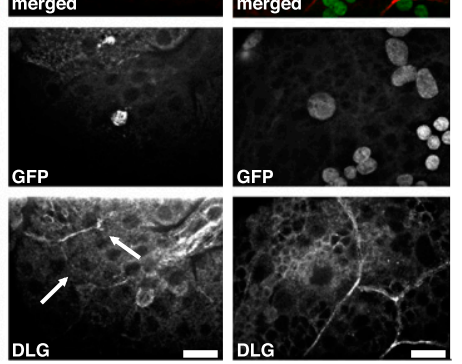

B UAS $>$ cdt1(dup)
UAS $>$ gfp

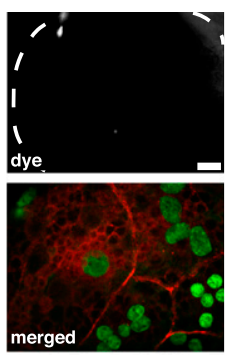

B
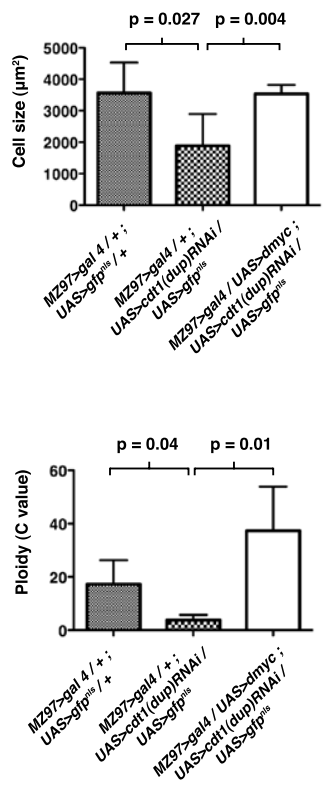

Figure 4. Polyploidy is necessary for a functional blood-brain barrier. $(A)$ Dye penetration was observed in $M Z 97>g a 14 /+; U A S>c d t 1$ (dup)RNAi/UAS $>g f p^{n 1 s}$, but this defect was rescued by overexpression of $d m y c$ in $M Z 97>g a 14 / U A S>d m y c ; U A S>c d t 1$ (dup)RNAi/UAS $>g f p^{n l s}$ animals. The abrogated septate junctions also were rescued by $d m y c$ induction in the SPG. The wild-type DLG pattern is shown in third instar larval brain from MZ97>gal4(II)/+; $U A S>g f p^{n l s} /+$ controls. This pattern was disrupted (arrows) when replication was blocked in $M Z 97>g a 14 /+; U A S>c d t 1$ (dup)RNAi/UAS-gfp ${ }^{\text {nts }}$. and $d m y c$ induction restored the Dlg pattern in $M Z 97>g a 14 / U A S>d m y c ; U A S>c d t 1$ (dup)RNAi/UAS $>g f p^{n l s}$ to the same pattern as in the control. GFP signal marks SPG nuclei. Bars, $10 \mu \mathrm{m} .(B)$ Reduction in SPG cell size and ploidy after ablation of $c d t 1$ (dup) $\left[M Z 97>\right.$ gal4/+;UAS $>c d t 1$ (dup)RNAi/UAS $\left.>g f p^{n l s}\right]$ compared with control $\left(M Z 97>g a 14 /+; U A S>g f p^{n l s} /+\right)$. Cell size and ploidy were rescued to control levels by $d m y c$ overexpression [MZ97>gal4(II)/UAS $\left.>d m y c ; U A S>c d t 1(d u p) R N A i / U A S>g f p^{n l s}\right]$. The probabilities of significant differences between the samples are shown.

Fig. 3). Together, these experiments show that polyploidization is required for a functional blood-brain barrier.

Rescue of the blood-brain barrier by increasing SPG ploidy or decreasing neuronal mass

Why does blocking polyploidization in the SPG result in failure of the blood-brain barrier? Our findings that inhibition of polyploidization resulted in defective septate junctions, together with our observation that an increase in SPG cell size is correlated with an increase in ploidy (Supplemental Fig. 2), led us to hypothesize that when DNA replication is blocked in SPG, they become prone to rupture caused by the pushing force of the increasing brain mass. To test this idea, we attempted to rescue the defective bloodbrain barrier by two approaches: (1) artificially forcing an increase in SPG cell size in cdt(dup)-ablated background to accommodate the growing number of neurons, and (2) attenuating the increase in neuron numbers to reduce the force that is exerted on the SPG by the growing brain.

To increase SPG size, we overexpressed the dmyc oncogene. Overexpression of $d m y c$ in endoreplicating cells results in increased ploidy, likely due to an accelerated G1/S progression, and cell size is coordinately increased (Johnston et al. 1999; Pierce et al. 2004). Myc recently has been shown to promote glial cell growth (Reddy and Irvine 2011). We found that overexpression of $d m y c$ specifically in the SPG caused an increased DAPI signal, consistent with increased ploidy (Supplemental Fig. 4). When $d m y c$ was overexpressed in $c d t 1$ (dup)-ablated SPG, the blood-brain barrier was restored, as evidenced by a block to dye penetration into the brain (Fig. 4A). The breaks in septate junctions seen in $c d t 1$ (dup)RNAi animals were no longer present when $d m y c$ was overexpressed (100\% of brains had intact NrxIV and DLG staining patterns; $n=33$ ) (Fig. 4A). The ability of ectopic dmyc to cause growth and polyploidy when cdt1(dup) is ablated most likely is due to a low residual level of $c d t 1$ (dup) following RNAi depletion. Consistent with this hypothesis, when cdt1(dup) was ablated more fully with the moody>gal4(II) driver, the bloodbrain barrier and septate junctions were not restored by $d m y c$ overexpression. Additionally, dmyc overexpression with the moody>gal4(II) driver did not increase SPG ploidy (data not shown). This increased ploidy in the rescued SPG is due to an increase in replication, as shown by increased BrdU incorporation in dmyc overexpressed, cdt1(dup)RNAi SPG compared with cdt1(dup)RNAi alone $(21 \%[n=85]$ to $1.9 \%[n=52]$, respectively) (Supplemental Fig. 5A). Consistent with these results, the small cell size observed after $c d t$ (dup)RNAi went into effect was rescued by $d m y c$ overexpression (Fig. 4B; Supplemental Fig. $5 \mathrm{~B})$. Thus, overexpression of $d m y c$ in cdt1(dup)-ablated SPG appears able to accelerate the G1/S progression of the SPG cells to increase ploidy and, in turn, cell size. These data together indicate that polyploidization is necessary to maintain the function of the blood-brain barrier and that this maintenance is achieved through increased cell size.

As a reciprocal test of the hypothesis that the SPG envelope needs to accommodate the increased volume from neuroblast divisions, we inhibited these divisions. Amino acid starvation has been shown to attenuate both endoreplication and the mitotic cycle in larvae, blocking neuronal division in the developing brain (Britton and Edgar 1998). To ask whether decreasing the neuronal mass in the brain could rescue the blood-brain barrier defect in larvae with $c d t 1$ (dup)-ablated SPG, we examined the blood-brain barrier in moody>gal4(II)/+;cdt1(dup)RNAi first instar larvae (30-40 h after egg laying [AEL]) fed for 48 $h$ with food with amino acids (Kankel food) or food lacking amino acids (20\% sucrose solution in $1 \times \mathrm{PBS})$. When compared with the cdt1(dup)-ablated larvae fed with Kankel food, amino acid starvation rescued both the dye penetration defect and septate junction pattern defects $(100 \%$ of brains with rescued NrxIV and DLG patterns; $n=58$ ) (Supplemental Table 3; Supplemental Fig. 6, respectively). We showed that amino acid starvation indeed inhibited DNA replication and proliferation of cells within the brain of the larvae with cdt1(dup) ablated in the SPG (Supplemental Fig. 7). It is unlikely that amino acid starvation could cause $c d t 1$ (dup)-ablated 
SPG to increase in size to rescue the blood-brain barrier, because amino acid starvation attenuates endoreplication, which is already inhibited in the SPG in this experiment (Britton and Edgar 1998). Therefore, these experiments implicate neuroblast divisions and increasing brain size as causes of the failure of the blood-brain barrier when polyploidization is blocked in the SPG.

\section{SPG increase ploidy in response to increasing brain lobe volume}

To test whether SPG polyploidize in response to growth of brain size, we used homozygous aur ${ }^{8839}$ mutants, which have increased brain volume at the wandering third instar larval stage (Wang et al. 2006). The increased size of the brain lobes in this mutant was associated with increased SPG ploidy (Fig. 5). Both the ploidy of mononucleate SPG and the number of nuclei in multinucleate SPG cells increased. The increased ploidy of SPG retained a functional blood-brain barrier despite the large neuronal mass. Only a small fraction of the aur A mutant brains had a defective DLG pattern or dye permeability $(10 \% ; n=80)$ compared with control $(0 \% ; n=64)$ (Supplemental Fig. 8A; Supplemental Table 3). aurA mutations are specific for mitotic cells, as they affect centrosome separation /Glover et al. 1995), and aurA ${ }^{8839}$ does not detectably alter the ploidy of the endocycling larval salivary gland (Supplemental Fig. 8B). These results suggest that the SPG can increase ploidy in response to increased neuronal mass, coordinating growth with the growing brain size to maintain the blood-brain barrier.

How different tissues coordinate their growth to form the final, properly sized organ is not well understood. SPG envelop neurons via septate junctions to create the bloodbrain barrier during Drosophila development (Stork et al. 2008; Hatan et al. 2011). This strategy creates a develop-
A

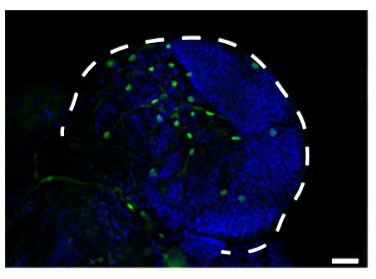

moody $>$ gal4/UAS $>$ GFP ${ }^{n / s} ;+/+$

B

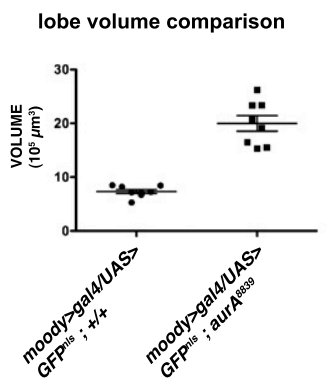

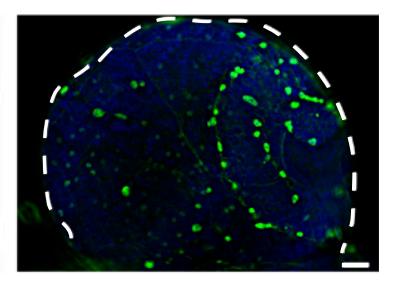

moody $>$ gal4/UAS $>G_{F P}$ n/s $;$ aurA $A^{839}$

C

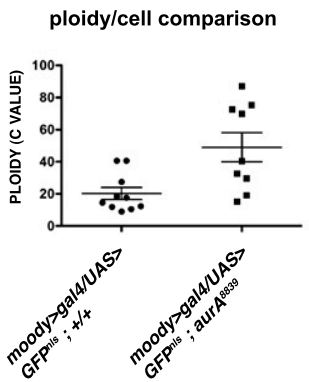

Figure 5. SPG ploidy increases with increasing brain volume. $(A)$ SPG are marked by moody $>$ gal4/uas $>G F P^{\text {nls }}$ in both control and the larger aurA mutant brain. Bars, $20 \mu \mathrm{m}$. Increased volume (in cubic microns) $(B)$ and ploidy per cell $(C)$ of mutant aur $A$ brains. White dashed lines outline the brain. The horizontal bars in $B$ and $C$ designate the mean ploidy and the standard deviation. mental conundrum, because neurons continue to divide and increase in mass, while SPG need to both maintain a tight seal and increase cell size to accommodate neuronal growth. Here we demonstrate that by increasing cell size via increases in ploidy, SPG can keep the cell number constant and maintain the septate junctions unperturbed by cytokinesis. Our experiments suggest that there exists feedback between the polyploidizing SPG tissue and the mitotic neuronal tissue to coordinate SPG ploidy with brain lobe size. Consistent with this model, the SPG of the brain lobes, which must cover a larger surface area than those in the ventral cord, attain a higher level of ploidy and cell size. It remains to be determined why some SPG are multinucleated and others have a single polyploid nucleus and why this is restricted to SPG in the brain. This study presents a novel mechanism for how different tissues are scaled to size in a developing brain, as well as establishing a new paradigm for the use of polyploidization to coordinate growth of different tissues during organogenesis.

The strategy of using polyploidization to coordinate tissue growth appears to be conserved in vertebrates and to function in other organ systems. Examples are the polyploidizing trophoblasts that surround the mitotic blastula during embryo implantation (Zybina and Zybina 2005), as well as the polyploid keratinocytes situated atop the dividing basal cell layer in the skin (Zanet et al. 2010). Both trophoblasts and keratinocytes form tight junctions. In trophoblasts, these are proposed to shield embryos from the potential harmful factors in the maternal environment (Wang et al. 2004), whereas in keratinocytes, the tight junctions provide a barrier for the skin epithelia (Brandner et al. 2006). Polyploidy is thus a likely strategy by which these polyploid tissues achieve growth while maintaining tight junctions. The signaling pathways these tissues and the SPG use for growth coordination remain to be defined. The coordination of SPG and brain growth in Drosophila reveals a mechanism of organogenesis that may have evolved to act also in vertebrate tissues with polyploid cells to control proper development of the tissue layers.

\section{Materials and methods}

\section{Fly strains}

The following fly strains were used in this study: 51252 (UAS>pcna RNAi) (VDRC), 23131 [UAS >cdt1(dup)RNAi] (VDRC), moody-gal4(II), moody> gal4(III), MZ97C (Beckervordersandforth et al. 2008; Stork et al. 2008), repo $>$ gal4 (M. Freeman, pers. comm.), H1::yfp (II) (Pinnola et al. 2007), $U A S>G F P^{n l s}$ (II and III), UAS $>d m y c$ (II) (Bloomington Stock Center), nrx::gfp (Banerjee et al. 2010), and aur ${ }^{8839}$ (Lee et al. 2006). In using the MZ97>gal4 driver line, SPG were examined solely in the VNC and brain lobes, as the driver (Beckervordersandforth et al. 2008) is not specific for SPG in the PNS.

\section{Cytological analysis and microscopy}

A detailed description for larval handling, antibody staining, EdU labeling, BrdU labeling, microscopy, DAPI quantification, cell size measurement, movie acquisition, and the dye penetration assay is in the Supplemental Material.

\section{FACS analysis}

Detailed descriptions of nuclear isolation from the larval brain and FACS are in the Supplemental Material. 


\section{Acknowledgments}

We are grateful to Christian Klämbt, Marc Freeman, Alexei Tulin, Manzoor Bhat, C.Y. Lee, and Mary Lou Pardue for Drosophila strains and reagents. We thank Marc Freeman for many helpful discussions and tutorials. We thank him as well as Michael Greenberg, Troy Littleton, and Peter Reddien for helpful comments on the manuscript. Tom DiCesare did the illustrations. This work was supported by the Harold and Leila Mathers Charitable Foundation and an American Cancer Society Research Professor award to T.O.-W.

\section{References}

Bainton RJ, Tsai LT, Schwabe T, DeSalvo M, Gaul U, Heberlein U. 2005. moody encodes two GPCRs that regulate cocaine behaviors and blood-brain barrier permeability in Drosophila. Cell 123: 145156.

Banerjee S, Bhat MA. 2007. Neuron-glial interactions in blood-brain barrier formation. Annu Rev Neurosci 30: 235-258.

Banerjee S, Blauth K, Peters K, Rogers SL, Fanning AS, Bhat MA. 2010. Drosophila neurexin IV interacts with Roundabout and is required for repulsive midline axon guidance. J Neurosci 30: 5653-5667.

Baumgartner S, Littleton JT, Broadie K, Bhat MA, Harbecke R, Lengyel JA, Chiquet-Ehrismann R, Prokop A, Bellen HJ. 1996. A Drosophila neurexin is required for septate junction and blood-nerve barrier formation and function. Cell 87: 1059-1068.

Beckervordersandforth RM, Rickert C, Altenhein B, Technau GM. 2008. Subtypes of glial cells in the Drosophila embryonic ventral nerve cord as related to lineage and gene expression. Mech Dev 125: 542-557.

Bell SP, Dutta A. 2002. DNA replication in eukaryotic cells. Annu Rev Biochem 71: 333-374.

Brandner JM, Kief S, Wladykowski E, Houdek P, Moll I. 2006. Tight junction proteins in the skin. Skin Pharmacol Physiol 19: 71-77.

Breuninger H, Lenhard M. 2010. Control of tissue and organ growth in plants. Curr Top Dev Biol 91: 185-220.

Britton JS, Edgar BA. 1998. Environmental control of the cell cycle in Drosophila: Nutrition activates mitotic and endoreplicative cells by distinct mechanisms. Development 125: 2149-2158.

Crickmore MA, Mann RS. 2008. The control of size in animals: Insights from selector genes. Bioessays 30: 843-853.

Desalvo MK, Mayer N, Mayer F, Bainton RJ. 2011. Physiologic and anatomic characterization of the brain surface glia barrier of Drosophila. Glia 59: 1322-1340.

Edenfeld G, Volohonsky G, Krukkert K, Naffin E, Lammel U, Grimm A, Engelen D, Reuveny A, Volk T, Klambt C. 2006. The splicing factor crooked neck associates with the RNA-binding protein HOW to control glial cell maturation in Drosophila. Neuron 52: 969-980.

Edgar BA, Orr-Weaver TL. 2001. Endoreplication cell cycles: More for less. Cell 105: 297-306.

Glover DM, Leibowitz MH, McLean DA, Parry H. 1995. Mutations in aurora prevent centrosome separation leading to the formation of monopolar spindles. Cell 81: 95-105.

Hatan M, Shinder V, Israeli D, Schnorrer F, Volk T. 2011. The Drosophila blood brain barrier is maintained by GPCR-dependent dynamic actin structures. J Cell Biol 192: 307-319.

Johnston LA, Prober DA, Edgar BA, Eisenman RN, Gallant P. 1999. Drosophila myc regulates cellular growth during development. Cell 98: 779-790.

Lee CY, Andersen RO, Cabernard C, Manning L, Tran KD, Lanskey MJ, Bashirullah A, Doe CQ. 2006. Drosophila Aurora-A kinase inhibits neuroblast self-renewal by regulating aPKC/Numb cortical polarity and spindle orientation. Genes Dev 20: 3464-3474.

Lee HO, Davidson JM, Duronio RJ. 2009. Endoreplication: Polyploidy with purpose. Genes Dev 23: 2461-2477.

Nagata Y, Muro Y, Todokoro K. 1997. Thrombopoietin-induced polyploidization of bone marrow megakaryocytes is due to a unique regulatory mechanism in late mitosis. J Cell Biol 139: 449-457.

Pierce SB, Yost C, Britton JS, Loo LW, Flynn EM, Edgar BA, Eisenman RN. 2004. $d M y c$ is required for larval growth and endoreplication in Drosophila. Development 131: 2317-2327.

Pinnola A, Naumova N, Shah M, Tulin AV. 2007. Nucleosomal core histones mediate dynamic regulation of poly(ADP-ribose) polymerase
1 protein binding to chromatin and induction of its enzymatic activity. J Biol Chem 282: 32511-32519.

Prokop A, Technau GM. 1991. The origin of postembryonic neuroblasts in the ventral nerve cord of Drosophila melanogaster. Development 111: 79-88.

Reddy BVVG, Irvine KD. 2011. Regulation of Drosophila glial cell proliferation by Merlin-Hippo signaling. Development 138: 5201-5212.

Robinow S, White K. 1991. Characterization and spatial distribution of the ELAV protein during Drosophila melanogaster development. J Neurobiol 22: 443-461.

Schwabe T, Bainton RJ, Fetter RD, Heberlein U, Gaul U. 2005. GPCR signaling is required for blood-brain barrier formation in Drosophila. Cell 123: 133-144.

Silies M, Yuva Y, Engelen D, Aho A, Stork T, Klambt C. 2007. Glial cell migration in the eye disc. J Neurosci 27: 13130-13139.

Smith AV, Orr-Weaver TL. 1991. Regulation of the cell cycle during Drosophila embryogenesis: The transition to polyteny. Development 112: 997-1008.

Stork T, Engelen D, Krudewig A, Silies M, Bainton RJ, Klambt C. 2008. Organization and function of the blood-brain barrier in Drosophila. I Neurosci 28: 587-597.

Strunnikov AV. 2010. One-hit wonders of genomic instability. Cell Div 5: 15. doi: 10.1186/1747-1028-5-15.

Styles JA. 1993. Measurement of ploidy and cell proliferation in the rodent liver. Environ Health Perspect 101: 67-71.

Technau GM. 2008. Brain development in Drosophila melanogaster. Landes Bioscience, Austin, TX, and Springer Science+Business Media, New York.

Wang X, Matsumoto H, Zhao X, Das SK, Paria BC. 2004. Embryonic signals direct the formation of tight junctional permeability barrier in the decidualizing stroma during embryo implantation. J Cell Sci 117: 53-62.

Wang H, Somers GW, Bashirullah A, Heberlein U, Yu F, Chia W. 2006. Aurora-A acts as a tumor suppressor and regulates self-renewal of Drosophila neuroblasts. Genes Dev 20: 3453-3463.

Whittaker AJ, Royzman I, Orr-Weaver TL. 2000. Drosophila DOUBLE PARKED: A conserved, essential replication protein that colocalizes with the origin recognition complex and links DNA replication with mitosis and the down-regulation of S phase transcripts. Genes Dev 14: $1765-1776$.

Woods DF, Bryant PJ. 1991. The discs-large tumor suppressor gene of Drosophila encodes a guanylate kinase homolog localized at septate junctions. Cell 66: 451-464.

Xiong WC, Okano H, Patel NH, Blendy JA, Montell C. 1994. repo encodes a glial-specific homeo domain protein required in the Drosophila nervous system. Genes Dev 8: 981-994.

Zanet J, Freije A, Ruiz M, Coulon V, Sanz JR, Chiesa J, Gandarillas A. 2010. A mitosis block links active cell cycle with human epidermal differentiation and results in endoreplication. PLOS ONE 5: e15701. doi: 10.1371/journal.pone.0015701.

Zybina TG, Zybina EV. 2005. Cell reproduction and genome multiplication in the proliferative and invasive trophoblast cell populations of mammalian placenta. Cell Biol Int 29: 1071-1083. 


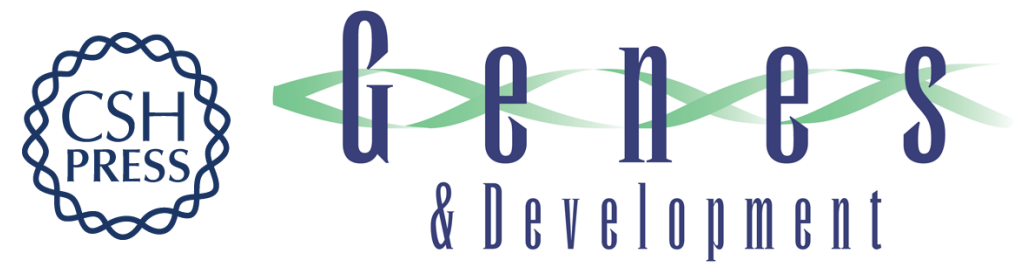

\section{Polyploidization of glia in neural development links tissue growth to blood-brain barrier integrity}

Yingdee Unhavaithaya and Terry L. Orr-Weaver

Genes Dev. 2012, 26:

Access the most recent version at doi:10.1101/gad.177436.111

Supplemental
Material http://genesdev.cshlp.org/content/suppl/2012/01/03/26.1.31.DC1

References This article cites 37 articles, 17 of which can be accessed free at: http://genesdev.cshlp.org/content/26/1/31.full.html\#ref-list-1

License Freely available online through the Genes \& Development Open Access option.

Email Alerting Receive free email alerts when new articles cite this article - sign up in the box at the top Service right corner of the article or click here.

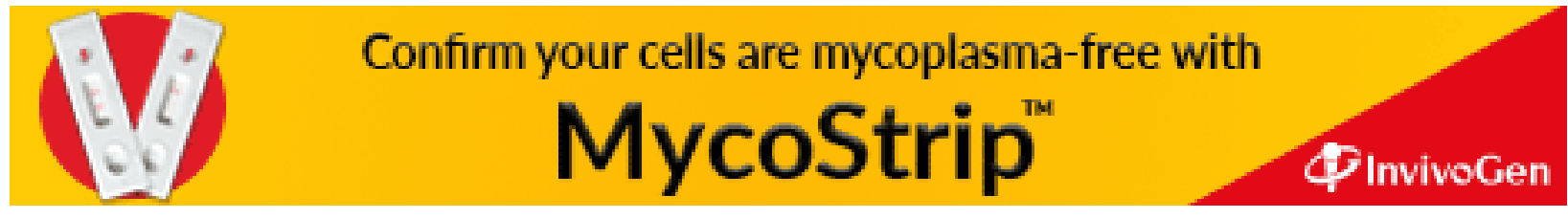

